\title{
RETRACTION
}

\section{Cellular and molecular immunopathogenesis of ulcerative colitis}

\author{
Suzhen Zhang ${ }^{1}$, Xuhui Zhao ${ }^{2}$ and Dechun Zhang ${ }^{1}$
}

Cellular \& Molecular Immunology (2014) 11, 314-314; doi:10.1038/cmi.2014.18; published online 24 March 2014

Retraction to: Cellular \& Molecular Immunology 2006; Volume 3, Number 1, page 35-40.

The reader of CMI has indicated to the journal that this paper exited some plagiarism and copy from other publications.

After a seriously investigate and analysis, a conclusion was drawn that this paper named: "Cellular and molecular immunopathogenesis of ulcerative colitis" is really a plagiarism paper. The editorial board and the editorial office of CMI feel sorry in fail of control the quality of the paper published in CMI. All manuscripts submitted by the listed authors in the plagiarism paper will be rejected without peer-review in future 5 years as a punishment.

The editorial office apologizes to the readers for any inconvenience caused.

\footnotetext{
${ }^{1}$ Department of Laboratory Medicine, Chongqing University of Medical Science, Chongqing, China and ${ }^{2}$ The Frist People's Hospital of Xingtai, Xingtai, China Correspondence: Dr SZ Zhang, Department of Laboratory Medicine, Chongqing University of Medical Science, Chongqing 400016, China.

E-mail: zhangsuzhen385@sohu.com
} 